\title{
Indice, signe et marque : exploration des liens synonymiques (DES) et configuration sémantique
}

Jean-Claude Dunkhorst et Caroline Masseron

\section{(2) OpenEdition}

12 Journals

Édition électronique

URL : https://journals.openedition.org/pratiques/1356

DOI : 10.4000/pratiques.1356

ISSN : 2425-2042

Éditeur

Centre de recherche sur les médiations (CREM)

Édition imprimée

Date de publication : 15 juin 2009

Pagination : 1-26

Référence électronique

Jean-Claude Dunkhorst et Caroline Masseron, «Indice, signe et marque : exploration des liens synonymiques (DES) et configuration sémantique », Pratiques [En ligne], 141-142 | 2009, mis en ligne le 20 juin 2014, consulté le 12 mars 2023. URL : http://journals.openedition.org/pratiques/1356 ; DOI : https://doi.org/10.4000/pratiques. 1356 


\title{
Indice, signe et marque : exploration des liens synonymiques (DES) et configuration sémantique
}

\section{Jean-Claude Dunkhorst, Caroline Masseron}

\author{
Université Paul-Verlaine, Metz, CELTED, EA 3474
}

\begin{abstract}
Marquer enchérit sur indiquer et sur désigner sous le rapport de la précision et de la certitude.

La marque fait reconnaître, l'indice met seulement sur la voie, et le signe donne seulement à entendre.

On donne à une personne des marques d'estime, et non pas seulement des indices ou des signes d'estime.

Lafaye 1870, Dictionnaires des synonymes de la langue française, p. 767.
\end{abstract}

L'article qu'on va lire est issu de deux projets complémentaires : le premier est un projet de thèse en didactique qui porte sur la notion d'indice, le second, une investigation méthodique du DES (Dictionnaire Électronique des Synonymes) qui ajoute à indice les items de signe et marque au titre des liens synonymiques existants et envisagés alors en sémantique lexicale. Nous voudrions dans une introduction succincte exposer la démarche et les objectifs des deux projets pour éclaircir une problématique qui sans doute ne va pas de soi, tant semblent éloignés les domaines et les méthodologies auxquels renvoient les projets. Ce sera pour nous l'occasion de faire le point sur les interrogations plus générales qui ne manquent pas de surgir, dans le champ de la recherche, au sujet des relations organiques qui associent - ou devraient, pourraient, associer - la didactique du français et la linguistique française. Auparavant, nous nous serons efforcés d'explorer, au moins sur la base des trois unités lexicales retenues, les dimensions sémantiques qui caractérisent des relations dites synonymiques ${ }^{(*)}$.

(*) Nous remercions chaleureusement Jacques François de sa relecture et des observations et propositions qu'il a faites. Les erreurs ou problèmes qui subsisteraient nous incombent évidemment. 


\section{Cadre général de la recherche engagée dans les deux domaines conjoints de la sémiotique narrative et de la didactique}

Le projet de thèse en didactique avait à l'origine l'intention d'investir les pratiques de lecture longue en classe sous l'angle des directives ministérielles de 2002 qui ont préconisé l'introduction de la « lecture littéraire » dans les classes de l'école élémentaire, et des situations d'enseignement qui ont dû dans ces conditions être mises en œuvre par les maîtres. Ce projet s'est donné deux champs d'application qui tous les deux convoquent une notion commune, celle d'indice. Tout d'abord, nous souhaitions procéder à une analyse de discours ayant pour objet la doxa didactique sur la construction du sens; pour ce faire, nous nous sommes enquis des occurrences de l'expression (prélever des) indices de lecture, fortement sollicitée, à la fois par les discours de la doxa et par les maîtres dont nous avons pu suivre les séances en classe. C'est ainsi que nous avons décelé un rapport de concurrence (de synonymie ?) entre l'expression indices de lecture avec l'usage non moins fréquent de l'expression (faire des) hypothèses de lecture. Il nous est paru qu'une telle analyse pouvait déboucher sur la clarification qui nous semblait nécessaire, au plan didactique, entre lecture et interprétation du texte.

Parallèlement, on ne s'étonnera pas que ce soit le genre du récit d'énigme policière qui ait servi à constituer le recueil de fictions littéraires destinées à la jeunesse ou aux adultes, dont nous avions besoin pour comprendre la fonction sémiotique des indices. Prélever les indices matériels et les interpréter entre dans les prérogatives de tout détective et l'on pouvait s'attendre à ce que les classiques du genre fassent un usage important de scènes se rapportant aux indices qui conduisent l'enquêteur au dévoilement de l'intrigue policière. À l'instar de Todorov (1971), des critiques comme J. Dubois (2006) ont identifié l'herméneutique du roman policier comme une forme de mise en abyme, cryptogramme ou métaphore de toute lecture : de l'interprétation, par l'enquêteur, du réel où est « cachée » la scène du crime au décodage du texte par le lecteur du roman policier, un lien métaphorique s'établit :

Toute enquête policière a pour objet la reconstitution d'une histoire passée. Histoire d'un crime que son auteur a eu intérêt à escamoter, dont il a tenu à effacer les traces. Par bonheur pour l'investigateur, il y a toujours ou presque, un reste. Quelques résidus, matériels ou non, de l'action première demeurent et il s'agit tout à la fois de les reconnaître et de les déchiffrer. À propos de ce travail de caractère herméneutique, deux images parmi d'autres peuvent être invoquées. L'une revient d'innombrables fois dans le discours littéraire policier : approximative, elle compare l'acte herméneutique à la reconstitution d'un puzzle. Elle voit le détective comme celui qui patiemment assemble les pièces d'une séquence passée jusqu'à ce que des fragments naissent un ensemble cohérent, complet et significatif. On lui préfèrera l'image qui compare les traces à une écriture et leur déchiffrement à une lecture. (Dubois $2006: 123$ )

Traces, résidus, pièces (du puzzle), fragments, les indices relaient les deux univers du crime puis de l'enquête, jusqu'à devenir les signes du texte lui-même lors de la lecture « intelligente » que l'amateur de romans policiers réalise sous la conduite de l'enquêteur et du narrateur. En d'autres termes, indice(s) est envi- 
sagé comme un élément partiel, plus ou moins matériel, la source qui conduit à l'activité cognitive et abstraite que serait l'acte interprétatif (du réel ou du texte). La matérialisation elle-même tient au réel ou au texte dans leur totalité, tous les deux des entités « concrètes » qui sont les supports formels de l'acte cognitif.

Rappelant la gradation de Lafaye qui figure en exergue (marquer $>$ indiquer $>$ désigner; les marques $>$ les indices $>$ les signes), notre entrée en matière sur les indices oppose un univers concret, marqué mais saisi partiellement, et une opération de raisonnement, abstraite, qui vise à « reconstituer » l'univers fragmenté. La médiation entre l'univers et le raisonnement est réalisée grâce aux « indices ».

\section{Le calcul des liens synonymiques de l'item indice avec signe, preuve et marque}

La préface de Robert Martin à l'ouvrage de Jacques François, Pour une cartographie de la polysémie verbale, souligne le caractère expérimental de la démarche linguistique entreprise et sa portée méthodologique (François 2007 : XI). De fait, l'usager ne lit pas cet ouvrage comme il lirait un quelconque opuscule de théorie linguistique, mais il le lit plutôt comme si c'était la notice d'utilisation, théorique et pratique, d'une machine complexe, pour s'entraîner lui-même à la mise en œuvre de calculs sophistiqués et à l'examen des résultats que livrent ces calculs. Le travail présenté ici même sur indices tient lieu d'exercice d'application.

Très globalement, on rappellera que le travail exposé s'appuie sur une base de données lexicales, le Dictionnaire Electronique des Synonymes (désormais $D E S$ ) du CRISCO, sur divers calculs informatiques et procédures qui par étapes traitent la proximité ou la distance (sémantique) des items sélectionnés, et sur la projection d'un espace sémantique, multidimensionnel, qui visualise les liens sémantiques ainsi élaborés.

L'outil DES fusionne, pour une même entrée, sous forme de listes, les synonymes extraits de sept dictionnaires : deux dictionnaires de langue, le Grand Larousse de la Langue Française (1971) et le Grand Dictionnaire Robert (1992), quatre dictionnaires de synonymes explicatifs, Guizot (1864) et Lafaye (1878) pour le XIX ${ }^{\mathrm{e}}$ siècle ; Bénac (1956) et Bailly (1947) pour le XX $\mathrm{X}^{\mathrm{e}}$ siècle. Le dictionnaire de synonymes non explicatif ${ }^{(1)}$ de Du Chazaud (1989) complète cette base de données qui atteint environ 45.000 entrées et qui forme un réseau de plus de 450.000 liens (François 2007 : 2). Nous signalons dans la bibliographie d'autres dictionnaires consultés manuellement ; nous souhaiterions également formuler des réserves ou des interrogations, concernant les données du $D E S$, que nous ne sommes pas parvenus à éclaircir nettement :

i) Le traitement statistique des renvois analogiques (V. pour « « voir»), des occurrences définitoires (par exemple, le mot signe figurant comme terme générique des définitions d'indice dans les dictionnaires Robert) vs la spé-

(1) C'est-à-dire sans que l'entrée fasse l'objet d'un article rédigé. 
cificité synonymique des items : par exemple ébauche sous l'entrée indice dans le Robert, vocables auxquels l'intuition linguistique conteste le lien de synonyme ${ }^{(2)}$.

ii) L'appréhension des phénomènes historiques : s'agissant d'une unité comme la nôtre, on peut s'attendre à ce que les dictionnaires les plus contemporains renforcent les usages récents les plus saillants (indice de pauvreté, indice du CAC 40 : "Chiffre»), qui sont absents des articles de Guizot ou Lafaye, lesquels pourront en revanche noter signalement ou signature qui ne figurent pas dans nos résultats. Un autre terme, enseigne, figurant cette fois dans les synonymes d'indice, il est assorti de la mention $v x$ par le Grand Robert.

iii) La neutralisation dans les calculs des phénomènes de «copie » d'un dictionnaire à l'autre et les effets de redondance ou, au contraire, d'effacement. Les reprises sont d'autant plus frappantes que les dictionnaires sont brefs et spécialisés. L'exemple des trois verbes, marquer, indiquer et désigner, cités en exergue, est une reprise stricte de Guizot : Lafaye en a notamment conservé l'ordre et la catégorie grammaticale.

Nous nous efforçons dans les lignes qui suivent d'expliciter les étapes méthodiques d'une démarche que nous souhaiterions prudente et modeste. La puissance de l'outil lui-même, le $D E S$, et les analyses que réussit à produire Jacques François à partir d'un usage très savant et scrupuleux du même $D E S$ nous inclineraient presque à renoncer, étant donné le difficile équilibre à trouver entre calculs informatiques et investigations linguistiques. Au moment du bilan, il conviendra d'évaluer le coût occasionné par la maîtrise de l'outil et les bénéfices que l'on peut en attendre dans le domaine de la sémantique lexicale.

Plus précisément, notre recherche sur indice s'écartera de celle de Jacques François sur les trois points suivants :

- Une entrée vedette identifiée, indice, choisie préalablement sur la base d'un intérêt pour la notion, sa fonction narrative dans le contexte identifié du roman policier, sa polysémie et son degré d'abstraction (supra),

- La catégorie nominale d'indice, qui oriente les observations syntaxiques selon des voies spécifiques (les déterminants, les adjectifs, l'opposition singulier/pluriel, les collocations en de $N$, etc.),

- Le statut respectif de la synonymie et de la polysémie dans leur rapport avec d'autres catégories sémantiques et lexicales, particulièrement ici les opérations d'abstraction et de quantification, l'hypéronymie et l'hyponymie.

Rappelons brièvement que François (2007) quant à lui sélectionne onze verbes « vedettes », à l'issue d'une sélection préalable et critériée ${ }^{(3)}$ (affecter, appe-

(2) À vrai dire, le lien synonymique signalé requiert apparemment que l'on inverse l'orientation : le lien part de l'entrée ébauche et conduit à indice. C'est ainsi en tout cas que l'extension figurée de la définition d'ébauche dans le Grand Robert utilise indice: "Premier indice, premier développement d'une chose. » Le TLF procède de même dans la partie B de son article ébauche.

(3) Le domaine conceptuel, le regroupement lexicographique ( $v s$ le dégroupement) et le complément prédicatif configurent, pour l'essentiel, une polysémie qu'on dira moyenne pour les vedettes retenues. 
ler, changer, charger, couper, manquer, monter, rapporter, relever, reposer, voler) et qu'il élabore pour chacun d'eux une synthèse dite " espace sémantique » (Chapitre III, 117-253) qui rend compte des étapes suivies. L'étude de chaque verbe rend compte 1. du profil dictionnairique, 2. du fléchage synonymique de la polysémie, et 3. des relations sémantiques et syntaxiques qui sont fondées sur l'analyse des "cadres participatifs " (François 2003). L'ouvrage s'achève justement sur des interrogations sémantaxiques (Chapitre IV, 255-298, « De la sémantique à la syntaxe : les aires sémantaxiques des cadres participatifs à complément prédicatif ») qui associent des propriétés sémantiques (aspect, structure argumentale) et syntaxiques (fonctions). Ce dernier chapitre s'appuie sur un corpus double, romanesque et journalistique.

\subsection{Liens (inter-)synonymiques, cliques et matrices}

La synonymie est comme on le sait une relation partielle : elle caractérise le fait qu'une occurrence d'unité commute avec l'occurrence d'une autre unité dans un cotexte identifié. Les deux unités sont alors considérées comme synonymes dans le cotexte quand le sens de l'énoncé n'a pas radicalement changé. Par exemple, Indice et marque dans le cotexte laisser des $N$ de son passage : laisser des indices (marques) de son passage. Au contraire, la substitution des deux mêmes unités indice et marque dans le cotexte un $N$ de confiance aboutit à dissocier deux emplois et deux significations : un indice de confiance et une marque de confiance, le premier renvoyant à la cotation boursière, la seconde interprétant un événement domestique quelconque.

Les relations intersynonymiques sont, comme le nom l'indique, les liens de synonymie entre les synonymes de l'entrée vedette, ici indice (François 2007 : 34). Mais la relation de synonymie n'est pas toujours transitive dans la mesure où une unité lexicale A peut commuter avec une unité $\mathrm{B}$ dans un certain cotexte et avec une autre unité $\mathrm{C}$ dans un cotexte différent sans que les deux unités, B et $\mathrm{C}$, soient nécessairement à leur tour synonymes entre elles. Cette propriété contribue à « isoler » certains items, c'est-à-dire à les sortir d'une série inter-synonymique pour les constituer en une classe (dite composante secondaire, François 2007 : $65^{(4)}$ ) à une seule unité (ci-dessous, par exemple grade).

Nous pensons que l'examen des relations entre les différents synonymes d'un même mot, indice dans notre cas, nous aidera à approcher le degré de polysémie de ce mot. Nous utilisons le DES comme un «outil de fouille de la polysémie », selon la formule de Jacques François (2007 : 34), destiné à explorer les dimensions sémantiques qui caractérisent les relations synonymiques entretenues par les unités lexicales retenues : indice, signe et marque.

(4) Précisons que cette composante est toujours le résultat d'un calcul automatique. 


\title{
2.1.1. Liste des 26 synonymes d'indice
}

Voici les synonymes, par ordre alphabétique, qui sont issus de la consultation du $D E S$ :

Annonce, argument, caractère, caractéristique, charge, chiffre, classificateur, critère, ébauche, enseigne, index, indicateur, indication, marque, paramètre, présage, présomption, preuve, raison, renseignement, repère, signal, signe, symptôme, témoignage, trace.

\subsubsection{Répartition en 25 cliques}

La recherche du mot indice dans le DES fait apparaître une liste de 25 cliques. Une clique est un sous-ensemble d'unités qui sont toutes synonymes les unes des autres ; dans cette mesure, une clique peut être considérée comme « une coordonnée de sens » (François 2005 : 89). Pour faciliter la lecture des cliques, nous soulignons en italique les occurrences de marque et signe qui sont co-présents (par exemple 1 et 2 ) ou exclusifs ( 3 ou 23 ).

\author{
Liste des cliques d'indice \\ $1:$ annonce, indication, indice, marque, signe \\ 2 : annonce, indice, marque, présage, signe \\ 3 : annonce, indice, présage, signal, signe \\ $4:$ argument, indice, preuve, témoignage \\ 5 : argument, indice, raison \\ 6 : caractère, caractéristique, indice, marque, signe \\ 7 : caractère, chiffre, indice, marque, signe \\ 8 : caractère, critère, indice, marque, signe \\ 9 : charge, indication, indice, preuve \\ 10 : charge, indice, preuve, témoignage \\ 11 : charge, indice, présomption \\ 12 : classificateur, indice \\ 13 : critère, indication, indice, marque, preuve, signe \\ 14 : critère, indice, marque, preuve, signe, témoignage \\ 15 : enseigne, indice, marque, preuve, signe \\ 16 : grade, indice \\ 17 : index, indice \\ 18 : indicateur, indice \\ 19 : indication, indice, renseignement \\ 20 : ébauche, indication, indice \\ 21 : indice, marque, preuve, témoignage, trace \\ 22 : indice, marque, présage, signe, symptôme \\ 23 : indice, marque, repère, trace \\ 24 : indice, paramètre \\ 25 : indice, présage, signal, signe, symptôme
}

L'observation comparée des séries 2 et 3 permet d'illustrer la notion de cli- $^{-}$ que: s'il existe des liens synonymiques entre toutes les unités de chacune des cliques, l'absence de lien entre marque et signal explique la dissociation en deux cliques distinctes : les cliques 2 et 3 se différencient en raison de la non synonymie de marque et signal. 
Par ailleurs, on observe l'association d'indice à un seul synonyme, comme c'est le cas dans les cliques 12 (classificateur), 16 (grade), 17 (index), 18 (indicateur) et 24 (paramètre).

Enfin, il convient de repérer que, contrairement à marque et signe déjà évoqués au titre de leur omniprésence, plusieurs synonymes n'apparaissent qu'une seule fois : c'est le cas par exemple de raison et ébauche. Il en résulte un classement fondé sur le nombre de cliques auxquelles appartiennent les synonymes. Ce classement, où nous mentionnons le nombre de cliques d'appartenance entre parenthèses, est le suivant :

Marque (11), signe (11), preuve (7), indication (5), présage (4), témoignage (4), annonce (3), caractère (3), charge (3), critère (3), signal (2), symptôme (2), trace (2), argument (2), grade (1), indicateur (1), paramètre (1), présomption (1), raison (1), renseignement (1), repère (1), caractéristique (1), index (1), chiffre (1), classificateur (1), enseigne (1), ébauche (1).

On n'est pas autrement surpris de constater que le haut degré de lien synonymique vaut pour les deux termes les plus généraux (marque, signe), que ce degré chute rapidement (grade et $s q$ ), tandis que les unités qui figurent en fin de classement se révèlent être les plus spécifiques. Dans le cas des génériques, l'intuition pallie aisément l'absence de contexte ; inversement, certaines valeurs spécifiques requièrent un contexte qui atteste de la synonymie (ébauche?). L'étape suivante, celle du regroupement par composantes, éclaircit la question dans la mesure où elle permet notamment de discriminer des synonymes robustes ou fragiles.

Le classement des synonymes, fondé sur le nombre de cliques auxquelles ils appartiennent, est obtenu grâce à la matrice d'appartenance dont le résultat est disponible sur le site du CRISCO et qui permet de savoir à combien de cliques appartient chaque synonyme d'une composante. C'est ce que nous étudions dans le paragraphe qui suit.

\subsubsection{Regroupement par composantes : une composante principale et des composantes connexes, des synonymes robustes et des synonymes fragiles}

Le résultat de la requête portant sur indice affiche six composantes connexes (Cc) qui dissocient l'ensemble des 26 synonymes d'indice en plusieurs groupes dans lesquels les synonymes sont tous accessibles par un lien ou une chaîne de liens intersynonymiques :

$\mathrm{Cc} 1$ : annonce, argument, caractère, caractéristique, charge, chiffre, critère, ébauche, enseigne, indication, marque, preuve, présage, présomption, raison, renseignement, repère, signal, signe, symptôme, témoignage, trace

$\mathrm{Cc} 2$ : classificateur

$\mathrm{Cc} 3$ : grade

$\mathrm{Cc} 4$ : index

Cc5 : indicateur

Cc6 : paramètre 
Les cinq composantes (2 à 6) qui ne présentent qu'un seul synonyme correspondent à des sens isolés qui, dans les dictionnaires sources, ne renvoient à aucun autre synonyme qu'indice.

On retrouve ces composantes, disposées en un tableau qui fractionne les synonymes selon le critère de leur " robustesse » ou de leur « fragilité ». Est jugé robuste le synonyme qui est mentionné par plus d'une source, fragile celui qui n'apparaît que dans une seule source (François $2007: 61$ ).

\begin{tabular}{|c|c|c|c|c|c|c|}
\hline & $\begin{array}{c}\text { Composante } \\
1 \\
\end{array}$ & \begin{tabular}{|c} 
Composante \\
2 \\
\end{tabular} & \begin{tabular}{|c} 
Composante \\
$\mathbf{3}$ \\
\end{tabular} & $\begin{array}{c}\text { Composante } \\
4\end{array}$ & $\begin{array}{c}\text { Composante } \\
5\end{array}$ & $\begin{array}{c}\text { Composante } \\
6 \\
\end{array}$ \\
\hline $\begin{array}{l}\text { SYN } \\
\text { robus- } \\
\text { tes }\end{array}$ & $\begin{array}{l}\text { Signe } \\
\text { Charge } \\
\text { Indication } \\
\text { Caractéristique } \\
\text { Marque } \\
\text { Preuve } \\
\text { Présomption } \\
\text { Renseignement } \\
\text { Symptôme } \\
\text { Trace } \\
\text { Annonce } \\
\text { Caractère } \\
\text { Témoignage }\end{array}$ & $\emptyset$ & Grade & $\varnothing$ & $\emptyset$ & $\emptyset$ \\
\hline \begin{tabular}{|l} 
SYN \\
fragiles
\end{tabular} & \begin{tabular}{|l} 
Chiffre \\
Critère \\
Enseigne \\
Présage \\
Raison \\
Repère \\
Signal \\
Ebauche \\
Argument
\end{tabular} & Classificateur & $\emptyset$ & Index & Indicateur & Paramètre \\
\hline
\end{tabular}

Tableau 1 : Composante principale, Cc1, et disqualification ${ }^{(5)}$ des composantes connexes $\mathrm{Cc} 2, \mathrm{Cc} 3, \mathrm{Cc} 4, \mathrm{Cc} 5, \mathrm{Cc} 6$ de l'item indice

Le tableau 1 rappelle que cinq composantes (2-6) sont issues de cliques isolées (à un seul lien) et que quatre d'entre elles $(\mathrm{Cc} 2, \mathrm{Cc} 4, \mathrm{Cc} 5, \mathrm{Cc} 6)$ ne présentent aucun synonyme robuste. Ce sont des arguments pour éliminer ces composantes et ne prendre en considération, dans les étapes suivantes, que les 22 (13 +9) unités synonymiques de la composante 1 .

Dans un premier temps, la matrice d'appartenance, qu'on fait figurer ci-dessous (tableau 2 page suivante), ne prend en compte que les synonymes robustes de la première composante ; le tableau à double entrée permet de croiser chaque item (la coordonnée horizontale des abscisses) et la série des cliques restantes (la colonne verticale des ordonnées), une fois qu'ont été retirées les cliques isolées. Le tableau renseigné ( 1 pour la présence, 0 pour l'absence) donne pour cha-

(5) La disqualification de Cc3, où grade est synonyme robuste, mérite cependant une explication supplémentaire. 


\begin{tabular}{|c|c|c|c|c|c|c|c|c|c|c|c|c|c|}
\hline $\begin{array}{l}\text { Synonymes robustes de la composante } \mathrm{Cc} 1 \rightarrow \\
\text { Liste des cliques de l'item vedette } \text { indice }\end{array}$ & 䓂 & 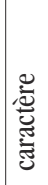 & 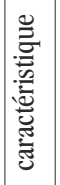 & 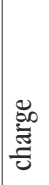 & 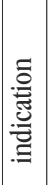 & 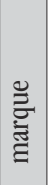 & 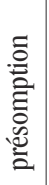 & 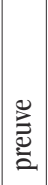 & 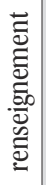 & 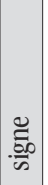 & 咅 & 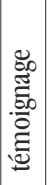 & $\underset{\mathbb{\Xi}}{\stackrel{\Xi}{\Xi}}$ \\
\hline 1 annonce, indication, indice, marque, signe & 1 & 0 & 0 & 0 & 1 & 1 & 0 & 0 & 0 & 1 & 0 & 0 & 0 \\
\hline 2 annonce, indice, marque, présage, signe & 1 & 0 & 0 & 0 & 0 & 1 & 0 & 0 & 0 & 1 & 0 & 0 & 0 \\
\hline 3 annonce, indice, présage, signal, signe & 1 & 0 & 0 & 0 & 0 & 0 & 0 & 0 & 0 & 1 & 0 & 0 & 0 \\
\hline 4 argument, indice, preuve, témoignage & 0 & 0 & 0 & 0 & 0 & 0 & 0 & 1 & 0 & 0 & 0 & 1 & 0 \\
\hline 5 argument, indice, raison & 0 & 0 & 0 & 0 & 0 & 0 & 0 & 0 & 0 & 0 & 0 & 0 & 0 \\
\hline 6 caractère, caractéristique, indice, marque, signe & 0 & 1 & 1 & 0 & 0 & 1 & 0 & 0 & 0 & 1 & 0 & 0 & 0 \\
\hline 7 caractère, chiffre, indice, marque, signe & 0 & 1 & 0 & 0 & 0 & 1 & 0 & 0 & 0 & 1 & 0 & 0 & 0 \\
\hline 8 caractère, critère, indice, marque, signe & 0 & 1 & 0 & 0 & 0 & 1 & 0 & 0 & 0 & 1 & 0 & 0 & 0 \\
\hline 9 charge, indication, indice, preuve & 0 & 0 & 0 & 1 & 1 & 0 & 0 & 1 & 0 & 0 & 0 & 0 & 0 \\
\hline 10 charge, indice, prewve, témoignage & 0 & 0 & 0 & 1 & 0 & 0 & 0 & 1 & 0 & 0 & 0 & 1 & 0 \\
\hline 11 charge, indice, présomption & 0 & 0 & 0 & 1 & 0 & 0 & 1 & 0 & 0 & 0 & 0 & 0 & 0 \\
\hline 13 critère, indication, indice, marque, preuve, signe & 0 & 0 & 0 & 0 & 1 & 1 & 0 & 1 & 0 & 1 & 0 & 0 & 0 \\
\hline 14 critère, indice, marque, preuve, signe, témoignage & 0 & 0 & 0 & 0 & 0 & 1 & 0 & 1 & 0 & 1 & 0 & 1 & 0 \\
\hline 15 enseigne, indice, marque, preuve, signe & 0 & 0 & 0 & 0 & 0 & 1 & 0 & 1 & 0 & 1 & 0 & 0 & 0 \\
\hline 19 indication, indice, renseignement & 0 & 0 & 0 & 0 & 1 & 0 & 0 & 0 & 1 & 0 & 0 & 0 & 0 \\
\hline 20 ébauche, indication, indice & 0 & 0 & 0 & 0 & 1 & 0 & 0 & 0 & 0 & 0 & 0 & 0 & 0 \\
\hline 21 indice, marque, prewve, témoignage, trace & 0 & 0 & 0 & 0 & 0 & 1 & 0 & 1 & 0 & 0 & 0 & 1 & 1 \\
\hline 22 indice, marque, présage, signe, symptôme & 0 & 0 & 0 & 0 & 0 & 1 & 0 & 0 & 0 & 1 & 1 & 0 & 0 \\
\hline 23 indice, marque, repère, trace & 0 & 0 & 0 & 0 & 0 & 1 & 0 & 0 & 0 & 0 & 0 & 0 & 1 \\
\hline 25 indice, présage, signal, signe, symptôme & 0 & 0 & 0 & 0 & 0 & 0 & 0 & 0 & 0 & 1 & 1 & 0 & 0 \\
\hline TOTAL & 3 & 3 & 1 & 3 & 5 & 11 & 1 & 7 & 1 & 11 & 2 & 4 & 2 \\
\hline
\end{tabular}

Tableau 2 : Matrice d'appartenance des synonymes robustes aux cliques de synonymes pour la composante connexe 1 de l'espace sémantique d'indice

que item un calcul du taux d'appartenance, qui rejoint celui auquel nous étions parvenus plus haut, si ce n'est la correction intervenue sur les synonymes fragiles ou isolés.

La matrice d'appartenance du tableau 2 vérifie que marque et signe apparaissent dans 11 cliques sur les 20 qui sont répertoriées ici. Ces deux unités présentent la couverture la plus vaste mais aussi des chevauchements importants :

- Marque et signe appartiennent en commun aux cliques 1, 2, 6, 7, 8, 13, 14, 15 , et 22 ,

- Marque appartient seul aux cliques 21 (preuve, trace, témoignage) et 23 (repère, trace),

- Signe appartient seul aux cliques 3 (annonce, présage et signal) et 25 (présage, signal et symptôme). 


\subsubsection{Synonymes et cliques extrêmes}

L'examen du tableau 2, et plus précisément la ligne inférieure des résultats, montre que trois synonymes, caractéristique, présomption et renseignement, n'apparaissent que dans une seule clique. Ils ont statut de synonyme " extrême » et leur clique de même est dénommée «clique extrême » :

- Clique 6 : caractère, caractéristique, indice, marque, signe

- Clique 11 : charge, indice, présomption

- Clique 19 : indication, indice, renseignement

Il faut ajouter cinq autres synonymes extrêmes, issus de la composante 1 mais au titre de synonymes fragiles : chiffre, enseigne, raison, repère, et ébauche. Partagent cette propriété les cliques auxquelles ils appartiennent:

- Clique 5 : argument, indice, raison

- Clique 7 : caractère, chiffre, indice, marque, signe

- Clique 14 : enseigne, indice, marque, preuve, signe

- Clique 16 : ébauche, indication, indice

- Clique 19 : indice, marque, repère, trace

\subsubsection{Matrice de coappartenance}

Le tableau qui suit (tableau 3 page suivante) enregistre, à partir des 22 synonymes «non isolés » dont l'entrée vedette indice, le nombre de cliques communes à l'item de l'abscisse et l'item de l'ordonnée. C'est ainsi que, dans le sousespace de la composante 1, annonce partage 2 cliques avec marque et 3 avec $s i$ gne (la première ligne du tableau). On dit alors (François $2007: 73$ ) qu'annonce a un indice de coappartenance aux cliques de 2 avec marque (mais aussi avec présage), et de 3 avec signe (mais aussi avec indice).

La diagonale du tableau neutralise l'impossibilité d'une coappartenance pour un même item (annonce avec annonce, etc.), mais le chiffre qui figure dans la case n'est autre que celui de la coappartenance avec l'item vedette indice. La matrice permet de retrouver le classement de la totalité des synonymes robustes et fragiles de la composante $\mathrm{Cc} 1$ en fonction du nombre de cliques : sur les 20 cliques possibles (voir l'intersection de la colonne et de la ligne correspondant à indice), marque et signe appartiennent respectivement à 11 cliques dont 9 communes, preuve à 7 cliques, indication à 5 , présage et témoignage à 4 , annonce, caractère, charge et critère à 3 , signal, symptôme et trace à 2 . On retrouve enfin la totalité des huit synonymes extrêmes (cases comportant le chiffre 1 dans la ligne ou la colonne correspondant à indice) : caractéristique, chiffre, ébauche, enseigne, présomption, raison, renseignement et repère.

Enfin, l'intérêt de cette matrice de coappartenance est de mettre en évidence un noyau de synonymes qui partagent un nombre important de cliques les uns avec les autres et en dehors de leurs liens avec la vedette. Les huit synonymes, retenus dans la figure 1, ont entre eux un indice de coappartenance supérieur ou égal à 3 :

- marque a un indice de coappartenance avec signe de 9,

- témoignage et preuve : 4, 


\begin{tabular}{|c|c|c|c|c|c|c|c|c|c|c|c|c|c|c|c|c|c|c|c|c|c|c|c|c|}
\hline $\begin{array}{l}\text { Fréquence } \\
\text { Liste des SYN }\end{array}$ & 造 & 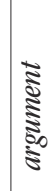 & $\frac{\sqrt{3}}{0}$ & 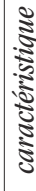 & $\frac{8}{3}$ & & 3 & בู & : & 离 & 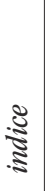 & 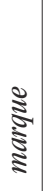 & 离 & 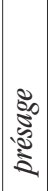 & 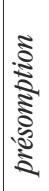 & 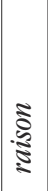 & 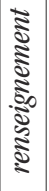 & 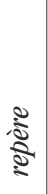 & 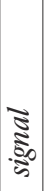 & . & 豙 & 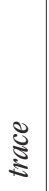 & 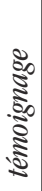 & $\begin{array}{l}2 \\
\frac{8}{3} \\
0 \\
0\end{array}$ \\
\hline annonce & 3 & 0 & 0 & 0 & 0 & 0 & & \begin{tabular}{l|l}
0 & 0 \\
0
\end{tabular} & 0 & 1 & 3 & 2 & 0 & 2 & 0 & 0 & 0 & 0 & 1 & 3 & 0 & 0 & 0 & 0 \\
\hline argument & & 2 & 0 & 0 & 0 & 0 & & $0 \quad 0$ & 0 & 0 & 2 & 0 & 1 & 0 & 0 & 1 & 0 & 0 & 0 & 0 & 0 & 0 & 1 & 0 \\
\hline caractère & & & 3 & 1 & 0 & 1 & & 10 & 0 & 0 & 3 & 3 & 0 & 0 & 0 & 0 & 0 & 0 & 0 & 3 & 0 & 0 & 0 & 0 \\
\hline caractéristique & & & & 1 & 0 & 0 & 0 & \begin{tabular}{l|l}
0 & 0 \\
\end{tabular} & 0 & 0 & 1 & 1 & 0 & 0 & 0 & 0 & 0 & 0 & 0 & 1 & 0 & 0 & 0 & 0 \\
\hline charge & & & & & 3 & 0 & & $0 \quad 0$ & 0 & 1 & 3 & 0 & 2 & 0 & 1 & 0 & 0 & 0 & 0 & 0 & 0 & 0 & 1 & 0 \\
\hline chiffre & & & & & & 1 & & \begin{tabular}{l|l}
0 & 0 \\
0
\end{tabular} & 0 & 0 & 1 & 1 & 0 & 0 & 0 & 0 & 0 & 0 & 0 & 1 & 0 & 0 & 0 & 0 \\
\hline critère & & & & & & & & 30 & 0 & 1 & 3 & 3 & 2 & 0 & 0 & 0 & 0 & 0 & 0 & 3 & 0 & 0 & 1 & 0 \\
\hline enseigne & & & & & & & & & 1 & 0 & 1 & 1 & 1 & 0 & 0 & 0 & 0 & 0 & 0 & 1 & 0 & 0 & 0 & 0 \\
\hline indication & & & & & & & & & & 5 & 5 & 2 & 2 & 0 & 0 & 0 & 1 & 0 & 0 & 2 & 0 & 0 & 0 & 1 \\
\hline indice & & & & & & & & & & & 20 & 11 & 7 & 4 & 1 & 1 & 1 & 1 & 2 & 11 & 2 & 2 & 4 & 1 \\
\hline marque & & & & & & & & & & & & 11 & 4 & 2 & 0 & 0 & 0 & 1 & 0 & 9 & 1 & 2 & 2 & 0 \\
\hline preuve & & & & & & & & & & & & & 7 & 0 & 0 & 0 & 0 & 0 & 0 & 3 & 0 & 1 & 4 & 0 \\
\hline présage & & & & & & & & & & & & & & 4 & 0 & 0 & 0 & 0 & 2 & 4 & 2 & 0 & 0 & 0 \\
\hline présomption & & & & & & & & & & & & & & & 1 & 0 & 0 & 0 & 0 & 0 & 0 & 0 & 0 & 0 \\
\hline raison & & & & & & & & & & & & & & & & 1 & 0 & 0 & 0 & 0 & 0 & 0 & 0 & 0 \\
\hline renseignement & & & & & & & & & & & & & & & & & 1 & 0 & 0 & 0 & 0 & 0 & 0 & 0 \\
\hline repère & & & & & & & & & & & & & & & & & & 1 & 0 & 0 & 0 & 1 & 0 & 0 \\
\hline signal & & & & & & & & & & & & & & & & & & & 2 & 2 & 1 & 0 & 0 & 0 \\
\hline signe & & & & & & & & & & & & & & & & & & & & 11 & 2 & 0 & 1 & 0 \\
\hline symptôme & & & & & & & & & & & & & & & & & & & & & 2 & 0 & 0 & 0 \\
\hline trace & & & & & & & & & & & & & & & & & & & & & & 2 & 1 & 0 \\
\hline témoignage & & & & & & & & & & & & & & & & & & & & & & & 4 & 0 \\
\hline ébauche & & & & & & & & & & & & & & & & & & & & & & & & 1 \\
\hline
\end{tabular}

Tableau 3 : Matrice de coappartenance des synonymes aux cliques des synonymes dans l'espace sémantique d'indice

- preuve et marque : 4,

- preuve et signe : 3 ,

- présage et signe : 4 ,

- caractère et marque : 3 ,

- caractère et signe : 3 ,

- critère et marque : 3 ,

- critère et signe : 3 ,

- annonce et signe: 3 .

Ces observations sont schématisées dans la figure 1 de la page suivante.

À vrai dire, la figure $1 \mathrm{n}$ 'apporte pas grand chose qu'on ne sache déjà (par exemple la position centrale de signe et marque) et, détail embarrassant, elle 


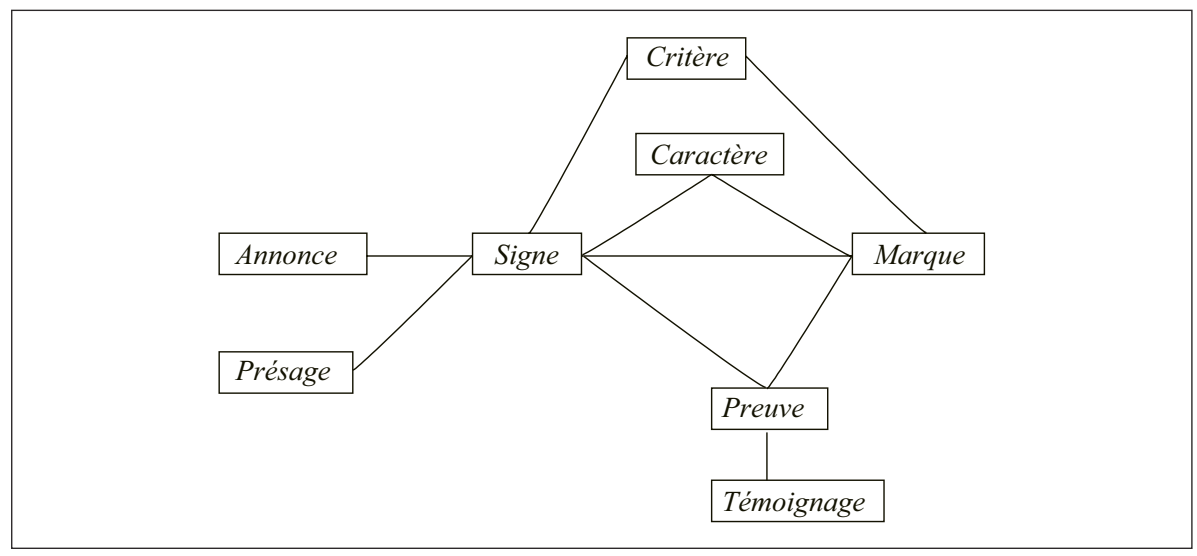

Figure 1 : Huit synonymes d'indice partageant entre eux trois cliques au moins

donne une place à caractère qu'un jugement linguistique spontané pourrait récuser ${ }^{(6)}$. Plus recevables en revanche sont les places occupées par annonce et présage (du côté de signe), et par preuve et témoignage, preuve faisant le lien entre témoignage et signe ou marque. Cette figure doit donc être lue avec prudence : elle est surtout là au titre d'un résultat d'étape, livraison brute mais sans doute encore hétérogène.

Au terme de cette partie, rappelons que nous avons principalement eu recours à divers données et outils du $D E S$ :

- Les 26 synonymes d'indice répartis en 25 cliques.

- Le tableau de source des liens synonymiques qui a permis de dissocier les synonymes fragiles des synonymes robustes.

- La liste des six composantes connexes de l'espace sémantique de la vedette indice qui provient en réalité de la matrice d'adjacence des synonymes que nous n'avons pas présentée.

- La matrice d'appartenance des synonymes (robustes) aux cliques de synonymes pour la composante connexe 1 de l'espace sémantique d'indice permettant d'évaluer la couverture de chacun des synonymes de cette composante. Cet outil a également permis d'identifier les synonymes extrêmes d'indice.

- La matrice de coappartenance des synonymes aux cliques des synonymes dans l'espace sémantique d'indice indique pour chaque synonyme le nombre de cliques qu'il partage avec indice. Cet outil nous a permis de mettre en évidence un noyau de six synonymes (annonce, caractère, critère, présage preuve, témoignage) qui partagent un nombre important de cliques avec signe et marque, les deux synonymes centraux d'indice.

L'utilisation des outils et les résultats auxquels nous parvenons, symbolisés par la figure 1 - tout anarchique qu'elle apparaisse -, auront, nous l'espérons,

(6) La polysémie du nom caractère n'inscrit pas l'acception «typographique » au premier rang de la typicalité. 
présenté l'intérêt d'expliciter des procédures d'élimination de synonymes extrêmes ou fragiles qui nous ont fait passer de 26 à 8 synonymes d'indice. Les calculs réalisés donnent le moyen de contrôler presque mécaniquement les procédures en question. Le plus difficile reste à faire : interpréter ces résultats statistiques et les faire correspondre à des données linguistiques, qu'il s'agisse de la signification en puissance d' indice ou de la compréhension du phénomène de «synonymie ».

\subsection{La construction des chaînes d'accès aux foyers de polysémie : fléchage, triplets, chaînes}

\subsubsection{Le fléchage conduisant aux foyers de polysémie, via les triplets proximaux}

Le fléchage (François 2007 : 75-80) est une voie d'accès aux synonymes centraux, les « foyers de polysémie ». La construction d'une chaîne d'accès repose sur le repérage d'un triplet proximal, soit l'unité lexicale vedette - ici nominale (N) - assortie de deux de ses synonymes. Le triplet proximal est ainsi formulé : $\{\mathrm{N}, \mathrm{SYNAlpha} \rightarrow$ SYNBéta $\}$.

Le SYNBéta (désormais SYN $\beta$ ) est le synonyme le plus proche de SYNalpha (désormais $\mathrm{SYN} \alpha$ ), c'est-à-dire le synonyme qui partage le plus de cliques avec SYN $\alpha$, et qui lui est superordonné (le SYN $\beta$ appartient à plus de cliques de la vedette que le $\mathrm{SYN} \alpha$ ). À l'inverse, le $\mathrm{SYN} \alpha$, appartenant à moins de cliques de la vedette que le SYN $\beta$, est qualifié de synonyme subordonné ou de synonyme $u l$ trapériphérique.

Une chaîne d'accès part donc d'un synonyme subordonné via un synonyme superordonné pour identifier un synonyme central que constitue le foyer de polysémie. Reste à présenter le mode de calcul utilisé qui permet d'obtenir le triplet proximal $\{\mathrm{N}, \mathrm{SYN} \alpha \rightarrow \mathrm{SYN} \beta\}$ et d'en décrire 1'orientation - 1'ordonner -, ce qui fonde l'accès au foyer de polysémie.

\subsubsection{Le calcul des triplets proximaux $\{\mathbf{N}, \mathrm{SYN} \alpha \rightarrow \mathrm{SYN} \beta\}$}

Il nous faut, pour obtenir ces différentes configurations de proximité synonymique, recourir à deux outils, la matrice de coappartenance des synonymes aux cliques (ci-dessus, le tableau 3 ), et la matrice de coappartenance différentielle (ci-dessous, les tableaux 4 et 6).

La coappartenance différentielle, comme son nom l'indique, procède à une comparaison du nombre de cliques d'appartenance pour une paire de synonymes donnés $\{\mathrm{SYN} \alpha, \mathrm{SYN} \beta$ \} et la vedette $(\mathrm{N})$. Le calcul donne une valeur positive si SYN $\alpha$ appartient à un plus grand nombre de cliques de synonymes que SYN $\beta$, une valeur négative dans le cas contraire, et une valeur nulle si les deux synonymes appartiennent à un nombre égal de cliques.

La matrice de coappartenance des synonymes aux cliques (Tableau 3 ) permet de déterminer quel sera pour chacun des SYN $\alpha$ d'un triplet proximal $\{\mathrm{N}, \mathrm{SYN} \alpha$ $\rightarrow \mathrm{SYN} \beta\}$ le SYN $\beta$. 
Considérons à titre d'exemple le triplet proximal \{indice, charge, SYN $\beta$ ?\} . Charge y est le SYNo. La consultation de la matrice de coappartenance des synonymes aux cliques (Tableau 3 ) permet de relever pour charge quatre synonymes partageant au moins une clique avec indice : indication, preuve, présomption et témoignage. Ils apparaissent avec leur indice de coappartenance respectif dans la première colonne du tableau ci-dessous. Sachant que charge a un indice de coappartenance de 3 avec indice (Tableau 3), il faut, pour obtenir les résultats de la coappartenance différentielle, soustraire ce chiffre de 3 à $l$ 'indice de coappartenance de chaque synonyme. L'indice de coappartenance figure également dans le tableau 3 : indication partageant 5 cliques avec indice a un indice de coappartenance (désormais, IDC) de 5, l'IDC de preuve est de 7, L'IDC de présomption et de témoignage équivaut à 1 . La coappartenance différentielle est de 2 pour indication : c'est le résultat de la soustraction entre les nombres de cliques des deux synonymes indication et charge, $5-3=2$.

\begin{tabular}{|l|c|c|}
\hline $\begin{array}{l}\text { Charge } \\
\text { IDC de 3 avec indice }\end{array}$ & $\begin{array}{c}\text { Coappartenance } \\
\text { brute }\end{array}$ & $\begin{array}{c}\text { Coappartenance } \\
\text { différentielle }\end{array}$ \\
\hline Indication & 1 & $5-3=2$ \\
\hline Preuve & 2 & $7-3=4$ \\
\hline Présomption & 1 & $1-3=-2$ \\
\hline Témoignage & 1 & $4-3=1$ \\
\hline
\end{tabular}

Tableau 4 : liste des synonymes d'indice partageant au moins une clique avec preuve

La valeur positive de 2 trouvée pour indication indique que le SYN $\beta$ (indication) appartient à plus de cliques de la vedette que le $\mathrm{SYN} \alpha$ (charge). À l'inverse, le nombre négatif de présomption signifie que le SYN $\beta$ (présomption) appartient à moins de cliques de la vedette que le SYN $\alpha$ (charge); présomption est par conséquent un synonyme subordonné au synonyme charge.

Après l'élimination de présomption, il reste à sélectionner comme synonyme superordonné (SYN $\beta$ ) l'un des trois synonymes que nous avons reproduits dans le tableau ci-dessous :

\begin{tabular}{|l|c|c|}
\hline Charge & $\begin{array}{c}\text { Coappartenance } \\
\text { brute }\end{array}$ & $\begin{array}{c}\text { Coappartenance } \\
\text { différentielle }\end{array}$ \\
\hline Indication & 1 & 2 \\
\hline Preuve & 2 & 4 \\
\hline Témoignage & 1 & 1 \\
\hline
\end{tabular}

Tableau 5 : Repérage du triplet proximal $\{\mathrm{N}=$ indice, $\mathrm{SYN} \alpha=$ charge $\rightarrow \mathrm{SYN} \beta=$ ? $\}$

Pour qualifier un synonyme de superordonné, François suggère les deux critères $(2007: 76)$ :

- Critère 1 : appartenir à plus de cliques de la vedette que le SYNo (ici, charge),

- Critère 2 : partager le plus grand nombre de cliques avec ce même SYN $\alpha$. 
L'application de ces deux critères permet de sélectionner le synonyme preuve.

Preuve en effet appartient à sept cliques contre trois pour le synonyme charge, d'où un plus grand différentiel, 4 (critère 1); il partage deux cliques avec le synonyme charge alors qu'indication et témoignage n'en partage qu'une seule (critère 2).

Il est donc désormais possible de constituer le triplet proximal $\{\mathrm{N}=$ indice, $\mathrm{SYN} \alpha=$ charge $\rightarrow \mathrm{SYN} \beta=$ preuve $\}$ qui délivre le maillon charge $\rightarrow$ preuve de la chaîne d'accès au foyer de polysémie.

Il s'agit à présent de déterminer si preuve, qui est un synonyme superordonné de charge, est lui-même le synonyme subordonné d'un autre SYN $\beta$. Pour cela nous renouvelons la méthode de construction des triplets proximaux en l'appliquant au triplet proximal \{indice, preuve, SYN $\beta$ ? \}. La consultation du tableau 3 permet de constater que la matrice de coappartenance délivre pour le SYN $\alpha$ preuve neuf synonymes partageant au moins une clique avec indice. Ils apparaissent avec leur indice de coappartenance respectif dans la première colonne du tableau ci-dessous. Sachant que preuve a un indice de coappartenance (IDC) de 7 avec indice, il suffit, pour obtenir les résultats de la coappartenance différentielle, de soustraire 7 à l'indice de coappartenance de chaque synonyme.

\begin{tabular}{|l|c|c|}
\hline $\begin{array}{l}\text { Preuve } \\
\text { IDC de 7 avec indice }\end{array}$ & $\begin{array}{c}\text { Coappartenance } \\
\text { brute }\end{array}$ & $\begin{array}{c}\text { Coappartenance } \\
\text { différentielle }\end{array}$ \\
\hline Argument & 1 & $2-7=-5$ \\
\hline Charge & 2 & $3-7=-4$ \\
\hline Critère & 2 & -4 \\
\hline Enseigne & 1 & -6 \\
\hline Indication & 2 & -2 \\
\hline Marque & 4 & 4 \\
\hline Signe & 3 & 4 \\
\hline Trace & 1 & -5 \\
\hline Témoignage & 4 & -3 \\
\hline
\end{tabular}

Tableau 6a: Liste des synonymes d'indice partageant au moins une clique avec preuve

La valeur négative (-5) de la coappartenance différentielle d'argument indique que l'item considéré, appartenant à moins de cliques de la vedette que le SYNo (preuve), est un synonyme subordonné. La matrice de coappartenance différentielle permet de repérer au total six synonymes subordonnés, appartenant à moins de cliques que preuve : ce sont argument, charge, critère, enseigne, indication, trace et témoignage.

Après l'élimination de ces derniers synonymes subordonnés, il reste à sélectionner comme SYN $\beta$ l'un des deux synonymes superordonnés : marque ou signe appartenant à plus de cliques de la vedette que le SYN $\alpha$ (preuve). 


\begin{tabular}{|l|c|c|}
\hline Preuve & $\begin{array}{c}\text { Coappartenance } \\
\text { brute }\end{array}$ & $\begin{array}{c}\text { Coappartenance } \\
\text { différentielle }\end{array}$ \\
\hline Marque & 4 & 4 \\
\hline Signe & 3 & 4 \\
\hline
\end{tabular}

Tableau $6 \boldsymbol{b}$ : Repérage dutriplet proximal $\{\mathrm{N}=$ indice, $\mathrm{SYN} \alpha=$ preuve $\rightarrow \mathrm{SYN} \beta=$ ?

On se trouve en présence d'un cas justifiant la nécessité de recourir aux deux critères mentionnés plus haut. Marque et signe satisfont en effet au premier critère : ils appartiennent tous deux à onze cliques, soit quatre de plus que preuve. En revanche marque partage quatre cliques avec preuve contrairement à signe qui n'en partage que trois. Il est donc possible de constituer un nouveau triplet proximal $\{\mathrm{N}=$ indice, $\mathrm{SYN} \alpha=$ preuve $\rightarrow \mathrm{SYN} \beta=$ marque $\}$ qui présente le maillon preuve $\rightarrow$ marque de la chaîne d'accès aux foyers de polysémie. En réitérant la procédure avec le synonyme présomption comme SYN $\alpha$, nous obtenons le maillon présomption $\rightarrow$ charge.

À ce stade, nous sommes en présence de trois maillons : le maillon (1) charge $\rightarrow$ preuve subordonné au maillon (2) preuve $\rightarrow$ marque, mais superordonné au maillon (3) présomption $\rightarrow$ charge. En combinant ces maillons, on obtient une chaîne d'accès au synonyme central marque, ce qui donne : présomption $\rightarrow$ charge $\rightarrow$ preuve $\rightarrow$ marque.

Dans cette chaîne, marque est un synonyme central ou foyer de polysémie, preuve est un synonyme périphérique de marque, charge un synonyme ultrapériphérique de rang 1 de preuve, et présomption un synonyme ultrapériphérique de rang 2 de charge. Le tableau 7 donne la liste des chaînes d'accès des synonymes de la vedette indice de la composante 1.

\begin{tabular}{|l|l|l|l|}
\hline $\begin{array}{l}\text { Synonyme } \\
\text { ultrapériphérique 2 }\end{array}$ & $\begin{array}{l}\text { Synonyme } \\
\text { ultrapériphérique1 }\end{array}$ & $\begin{array}{l}\text { Synonyme } \\
\text { périphérique }\end{array}$ & Foyer de Polysémie \\
\hline $\begin{array}{l}\text { Présomption } \\
\text { Raison }\end{array}$ & $\begin{array}{l}\text { Charge } \\
\text { Argument } \\
\text { Témoignage }\end{array}$ & $\begin{array}{l}\leftarrow \text { Preuve } \\
\leftarrow \text { Preuve } \\
\leftarrow \text { Preuve } \\
\text { Repère } \\
\text { Trace }\end{array}$ & Marque \\
\hline & $\begin{array}{l}\text { Renseignement } \\
\text { Ebauche }\end{array}$ & $\begin{array}{l}\leftarrow \text { Indication } \\
\leftarrow \text { Indication } \\
\text { Critère } \\
\text { Chiffre } \\
\text { Enseigne } \\
\text { Caractère } \\
\text { Caractéristique }\end{array}$ & Marque/Signe \\
\hline & $\begin{array}{l}\text { Présage } \\
\text { Symptôme } \\
\text { Signal } \\
\text { Annonce }\end{array}$ & Signe \\
\hline & & \\
\hline
\end{tabular}

Tableau 7 : Les chaînes d'accès aux foyers de polysémie de l'item indice 
Le tableau 7 fait apparaître que les vingt synonymes d'indice se répartissent autour de trois foyers de polysémie : le foyer de polysémie marque qui regroupe huit synonymes, le foyer de polysémie signe qui en compte quatre, et le foyer à deux composants marque et signe qui regroupe également huit synonymes.

Jacques François (2007 : 80-82) fait observer que certaines chaînes d'accès aux foyers de polysémie peuvent nécessiter des corrections en raison de leur hétérogénéité. De fait, les deux premières chaînes (1. présomption $\rightarrow$ charge $\rightarrow$ preuve $\rightarrow$ marque ; et 2 . raison $\rightarrow$ argument $\rightarrow$ preuve $\rightarrow$ marque) ne sont pas homogènes entre elles dans la mesure où charge ne partage aucune clique avec argument, ces deux synonymes n'étant reliés que par indice. Argument partage en revanche une clique avec preuve et témoignage: argument, indice, preuve, témoignage.

Un autre argument plaide en faveur de l'hétérogénéité des deux premières chaînes d'accès au foyer de polysémie marque : les synonymes ultrapériphériques (présomption et charge) ainsi que le synonyme ultrapériphérique raison des deux premières chaînes qui comportent chacune quatre maillons ne partagent aucune clique avec le foyer de polysémie marque.

Nous proposons, dans ces conditions, de valoriser le lien étroit entre preuve et marque, lien commun à quatre cliques, en segmentant la chaîne au-delà de preuve ; cette solution revient à considérer, dans les deux premières chaînes, preuve comme un nouveau foyer de polysémie auquel seraient rattachés les synonymes présomption et charge d'une part et raison et argument d'autre part.

Une telle solution présente l'avantage de réduire à trois le nombre de maillons de la chaîne créée preuve $\rightarrow$ marque $\rightarrow$ indice. Jacques François (2007 : 115) recommande lui-même de limiter une chaîne d'accès à trois maillons et de procéder à l'examen critique des synonymes ultrapériphériques (ici, charge, présomption, raison et argument) qui ne partagent pas de clique avec le foyer de polysémie. Nous suivrons cette piste, renonçant aux synonymes ultrapériphériques qui ne partagent aucune clique avec le foyer de polysémie marque, et conservant en revanche le synonyme ultrapériphérique témoignage de la troisième chaîne (témoignage, preuve, marque, indice) qui partage une clique avec marque.

Au terme de cette correction, les synonymes d'indice se répartissent autour de quatre foyers de polysémie ( $c f$. tableau 8 page suivante).

Un faible nombre (4) de foyers de polysémie résulte d'indice. Marque et signe présents tous deux dans onze cliques ont sans doute joué un rôle d'attracteur avec le risque de masquer d'autres foyers polysémiques potentiels. Une correction, opérée à la suite de la vérification des liens synonymiques, nous a conduits à promouvoir preuve comme troisième foyer de polysémie ${ }^{(7)}$.

Cette dernière étape, avant de parvenir à une discussion plus spécifiquement sémantique, a permis de dégager quatre foyers de polysémie pour l'item indice. La série obtenue toutefois n'offre pas toutes les garanties d'homogénéité : fautil y laisser preuve qui apparaît comme le synonyme le plus faiblement «polysé-

(7) Preuve apparaît, dans le tableau 8, à deux emplacements : il est le périphérique de marque, via témoignage, et le foyer de polysémie pour charge et argument. 


\begin{tabular}{|l|l|l|l|}
\hline $\begin{array}{l}\text { Synonyme } \\
\text { ultrapériphérique 2 }\end{array}$ & $\begin{array}{l}\text { Synonyme } \\
\text { ultrapériphérique1 }\end{array}$ & $\begin{array}{l}\text { Synonyme } \\
\text { périphérique }\end{array}$ & Foyer de Polysémie \\
\hline & $\begin{array}{l}\text { Présomption } \\
\text { Raison }\end{array}$ & $\begin{array}{l}\text { Charge } \\
\text { Argument }\end{array}$ & Preuve \\
\hline & $\begin{array}{l}\text { Repère } \\
\text { Trace } \\
\text { Preuve }\end{array}$ & Marque \\
\hline & $\begin{array}{l}\text { Présage } \\
\text { Symptôme } \\
\text { Signal } \\
\text { Annonce }\end{array}$ & Signe \\
\hline & $\begin{array}{l}\text { Indication } \\
\text { Indication }\end{array}$ & Marque / Signe \\
Renseignement & $\begin{array}{l}\text { Critère } \\
\text { Chiffre } \\
\text { Enseigne } \\
\text { Caractère } \\
\text { Caractéristique }\end{array}$ & \\
& & & \\
\hline
\end{tabular}

Tableau 8 : Les chaînes d'accès aux foyers de polysémie de l'item indice

mique » ? et que penser du binôme marque / signe, relié aux synonymes renseignement, ébauche, indication, critère, chiffre, enseigne, caractère et caractéristique?

\section{Espace sémantique : foyers de polysémie et relations de synonymie}

\subsection{Elaboration des arbres de spécification sémantique}

Un « arbre de spécification sémantique », construit à l'aide des chaînes d'accès aux foyers de polysémies, n'est pas un outil du DES, sa conception et sa méthodologie reviennent à Jacques François (2007). Comme son nom l'indique, la spécification sémantique a pour objet d'atténuer la dispersion polysémique et, pour l'obtenir, 1'auteur inverse l'orientation du fléchage, ayant préalablement limité le nombre de maillons des chaînes (2007 : 85-92). Nous éprouvons ci-dessous la méthode sur trois foyers d'indice, successivement preuve, marque et $s i$ gne. Ce faisant, nous espérons trouver des éléments de réponse aux questions que soulèvent preuve et le binôme marque / signe.

\subsubsection{Premier arbre de spécification d'indice : preuve}

Les chaînes d'accès au foyer de polysémie preuve sont signalées dans le tableau $8:$ présomption $\rightarrow$ charge $\rightarrow$ preuve et raison $\rightarrow$ argument $\rightarrow$ preuve ; 1 'inversion des chaînes donne le résultat suivant : 
Preuve $\rightarrow$ charge $\rightarrow$ présomption

Preuve $\rightarrow$ argument $\rightarrow$ raison

\begin{tabular}{|c|c|c|c|}
\hline \multirow{2}{*}{ 点 } & \multirow{2}{*}{ 胥 } & $\leftarrow$ charge & $\leftarrow$ présomption \\
\hline & & $\leftarrow$ argument & $\leftarrow$ raison \\
\hline
\end{tabular}

Figure 2 : Preuve, extrait de l'arbre de spécification sémantique d'indice

La partie supérieure de l'arbre de spécification sémantique correspond partiellement à la rubrique I.2 que le Nouveau Petit Robert (2007) consacre à l'article indice :

\section{INDICE, 1.2}

Fait connu qui sert à constituer la preuve par présomption, début de preuve > présomption. La police n'a aucun indice.

Charge a une dispersion polysémique importante : la charge ou le chargement des objets à transporter, une charge de travail, autant d'acceptions disqualifiées par la relation avec présomption et preuve qui cantonne charge à son emploi judiciaire.

Considérons à présent la seconde chaîne de la figure $1:$ Preuve $\rightarrow$ argument $\rightarrow$ raison dans laquelle la dispersion polysémique du synonyme ultrapériphérique raison engendre un facteur d'hétérogénéité. Raison ne partage aucune clique avec preuve, n'entretenant de relation avec ce foyer de polysémie qu'à travers le synonyme argument.

\subsubsection{Second arbre de spécification sémantique d'indice : marque}

Le second sous-arbre qui regroupe cinq synonymes est rassemblé autour du foyer de polysémie marque, auquel donnent accès les trois synonymes périphériques repère, trace et preuve.

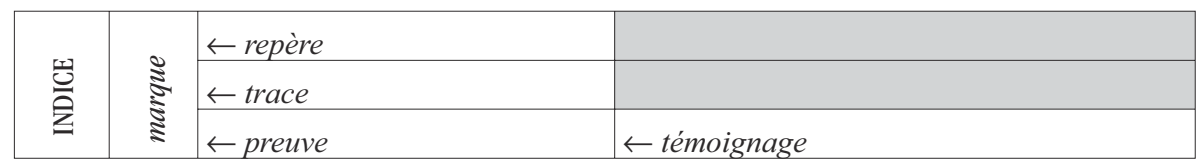

Figure 3 : Marque, extrait de l'arbre de spécification sémantique d'indice

Le synonyme ultrapériphérique témoignage entretient, à l'inverse des synonymes de même rang présomption et raison (figure 2), des liens avec marque ce dont atteste la commutation un(e) témoignage (marque) d'amitié, les deux synonymes appartenant aux cliques 21 (indice, marque, preuve, trace, témoignage) et 14 (critère, indice, marque, preuve, signe, témoignage).

Trace et repère synonymes périphériques de marque sont, directement ou indirectement, sollicités par le NPR sous l'entrée marque : 


\section{MARQUE, I.1}

Signe matériel, empreinte faite sur une chose pour la distinguer, la reconnaître ou pour servir de repère. $>$ Empreinte, signe.

On aura noté la présence d'empreinte et de signe à deux reprises (dans la définition et le renvoi analogique), qui ne figurait pas dans la série initiale des 26 synonymes d'indice. On pouvait déjà observer ce phénomène, ci-dessus, sous l'entrée indice dont la définition et le renvoi ont recours à preuve et présomption.

\subsubsection{Troisième arbre de spécification sémantique d'indice : signe}

Le dernier sous-arbre qui comporte cinq synonymes est rassemblé autour du foyer de polysémie signe auquel les quatre synonymes périphériques : présage, symptôme, signal et annonce donnent accès.

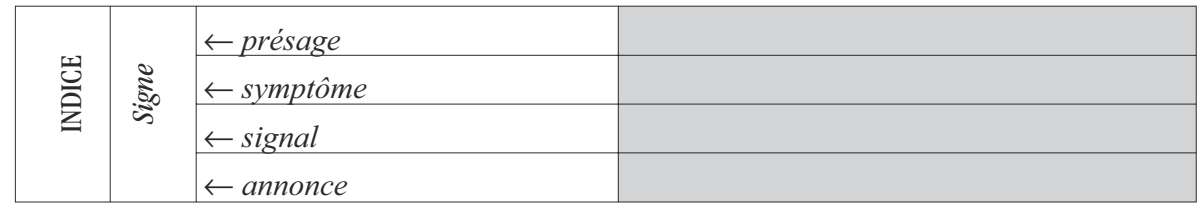

Figure 4 : Signe, extrait de 1'arbre de spécification sémantique d'indice

Les quatre chaînes d'accès au foyer de polysémie signe sont plutôt homogènes et l'on peut envisager les items présage, symptôme, signal et annonce comme des co-hyponymes de signe; le sous-arbre formé correspond à la rubrique I1 de l'article indice du NPR de laquelle on infère la co-hyponymie d'indice sous le même générique signe:

\section{INDICE, I1}

Signe apparent qui indique avec probabilité ce à quoi il est lié.

On comparera cette définition à celle de Mel'cuk et Polguère (2007) : « indication donnée par quelque chose ». Dans les deux cas est attesté le rôle prédicatif de l'indice.

\subsection{Discussion}

\subsubsection{Espace de visualisation}

Le $D E S$ offre la possibilité de représenter visuellement l'espace sémantique d'un item à partir d'un calcul savant des distances entre les cliques de synonymes. Toutefois, étant donné la multi-dimensionnalité des espaces sémantiques, une représentation bidimensionnelle, en un seul plan, nécessite de sélectionner les composantes sémantiques principales. Par conséquent, il faut se souvenir 
que les représentations obtenues ne sont que des raccourcis incomplets, et par certains côtés illusoires, de l'espace sémantique duquel elles sont issues. Ces réserves faites, voici une visualisation de l'espace sémantique d'indice sur le plan de projection $1 \times 2$ :

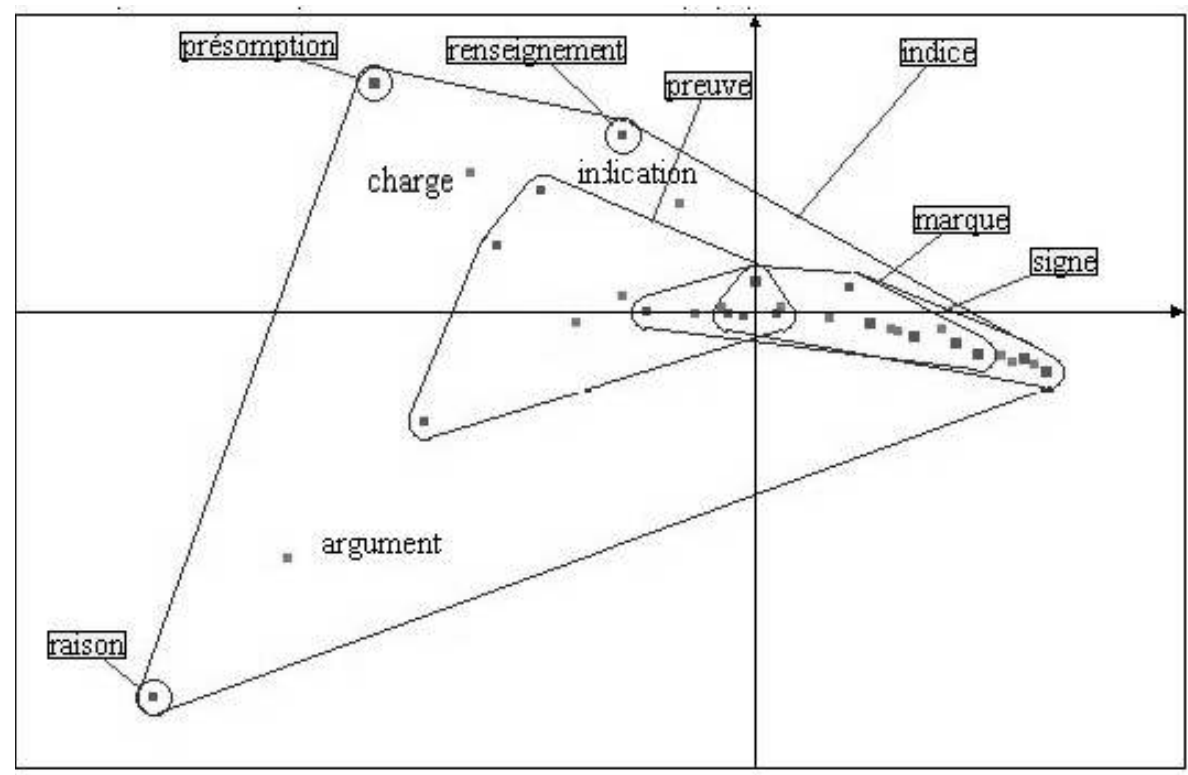

Figure 5 : Les trois foyers de polysémie (preuve, marque et signe) dans l'espace sémantique de la vedette indice sur le plan de projection $1 \times 2$

Les espaces correspondant aux trois foyers de polysémie : preuve, marque et signe englobent l'essentiel des 22 synonymes de la composante principale d'indice à l'exception d'argument, de charge et d'indication.

Les zones de chevauchement sont importantes comme le prévoyait le nombre élevé de cliques communes entre les foyers preuve et marque (4), preuve et signe (3) mais surtout marque et signe (9). L'intersection des trois foyers, quasiment à la croisée des deux axes à proximité du point correspondant à indice correspond aux cliques 13, 14 et 15 fortement tuilées :

- Clique 13 : critère, indication, indice, marque, preuve, signe

- Clique 14 : critère, indice, marque, preuve, signe, témoignage

- Clique 15 : enseigne, indice, marque, preuve, signe

Après l'exclusion des 3 cliques 13, 14 et 15, déjà mentionnées, on observe que la zone de chevauchement entre marque et signe demeure importante. Elle regroupe les synonymes chiffre et caractéristique dont les espaces sont quasiment superposés ; mais probablement s'agit-il d'un biais de la projection, caractéristique ne partageant aucune clique avec chiffre. 
Trois synonymes extrêmes n'appartenant qu'à une seule clique et correspondant à des sens précis d'indice, couvrent un espace limité et se situent aux extrémités de la représentation :

- Présomption, synonyme extrême robuste apparaît au sommet supérieur gauche du polygone représentant l'espace sémantique d'indice,

- Renseignement, synonyme extrême robuste correspond à un sommet situé dans une partie supérieure plus médiane,

- Raison, synonyme extrême fragile apparaît au sommet inférieur gauche du polygone.

\subsubsection{Le signifié de puissance et les synonymes d'indice. Synonymie, polysémie et généricité}

Comment traiter et synthétiser les informations recueillies jusque-là sur indice et ses synonymes ? De proche en proche, nous avons été conduits à identifier quatre foyers de polysémie : preuve, signe, marque et signe/marque. Nous en proposons la représentation figurée suivante, nous inspirant pour cela de la première tentative (figure 1) à laquelle nous reprochions un certain éclectisme :

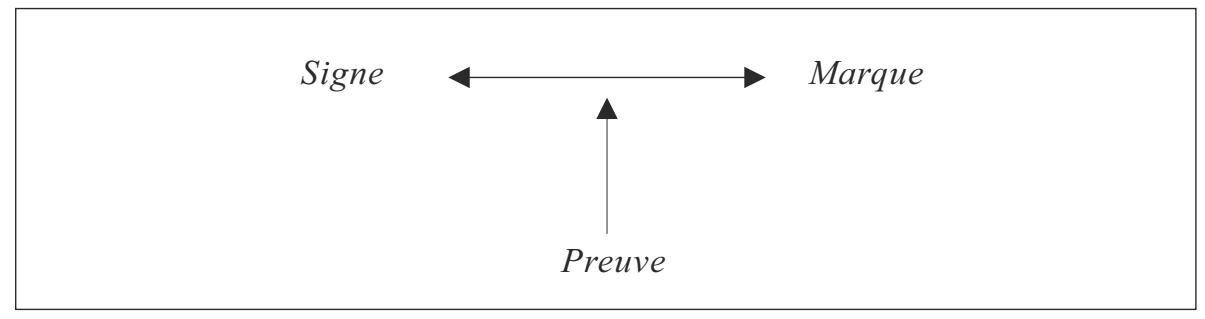

Figure 6 : foyers de polysémie d'indice

La double orientation horizontale qui conjoint signe et marque, fixe le noyau de sens principal d'indice, son « signifié de puissance » (Picoche 1986: 7-11). La simplicité de cette représentation schématique permet de déceler une dichotomie sémantique fondamentale, qui est celle de l'homme et du monde (l'univers physique et 1'univers du langage, Lowe 2007 : 21-46) ou, dit autrement, celle de l'agent interprétant (signe) et de l'objet donné, interprétable (marque). Le pôle de l'interprétant (signe) est investi du rôle cognitif de « comprendre et raisonner » (démontrer) ce que le pôle du réel observable a livré comme « donnée attestée » (ce qui est montré).

La position médiane et inférieure occupée par preuve renvoie à l'idée qu'une preuve réunit les deux opérations d'attestation (marque) et d'interprétation ( $s i^{-}$ gne), lesquelles portent plutôt sur des agissements humains, directement (la preuve qu'il est venu) ou non (la preuve que la porte n'était pas fermée). Preuve actualise nettement une idée d'orientation : la preuve va dans le sens d'une conclusion visée et implique un raisonnement un tant soit peu complet. Si l'hypothèse a quelque valeur, elle rappelle alors que preuve partage avec signe et marque la propriété d'être une unité singulière, partielle (« incomplétude ", "élé- 
ment distinct d'une pluralité homogène ou non »), et dont l'appréhension cognitive nécessite un préalable perceptif (visuel, olfactif, auditif, etc.).

Nous sommes en mesure de compléter la schématisation précédente et de lui ajouter les quelques fondements définitoires utiles :

\begin{tabular}{|l|l|}
$\begin{array}{l}\text { Perspective cognitive } \\
\text { (Interprétant) }\end{array}$ & $\begin{array}{l}\text { Donnée perceptible } \\
\text { (attestée, observable) }\end{array}$ \\
$\begin{array}{l}\text { Association intellective } \\
\text { (non aléatoire) d'une forme } \\
\text { et d'un sens possible }\end{array}$ & Marque
\end{tabular}

Figure 7 : le signifié de puissance d'indice d'après les définitions de ses synonymes centraux

La figure qu'on vient de lire (Figure 7) explique en partie pourquoi preuve n'a pas été retenu dans le titre de cet article. Son élimination tient à sa spécialisation supérieure, comparé à signe et marque qui sont au contraire remarquables pour leur généricité ; laquelle généricité explique aussi bien leur domination écrasante (cliques et coappartenance) que leur souplesse, peu discriminante, dès lors que l'on s'avise de les faire commuter avec tel ou tel item.

Enfin, voici, augmentés de leurs synonymes, les trois termes à partir desquels peut se conceptualiser la notion d'indice :

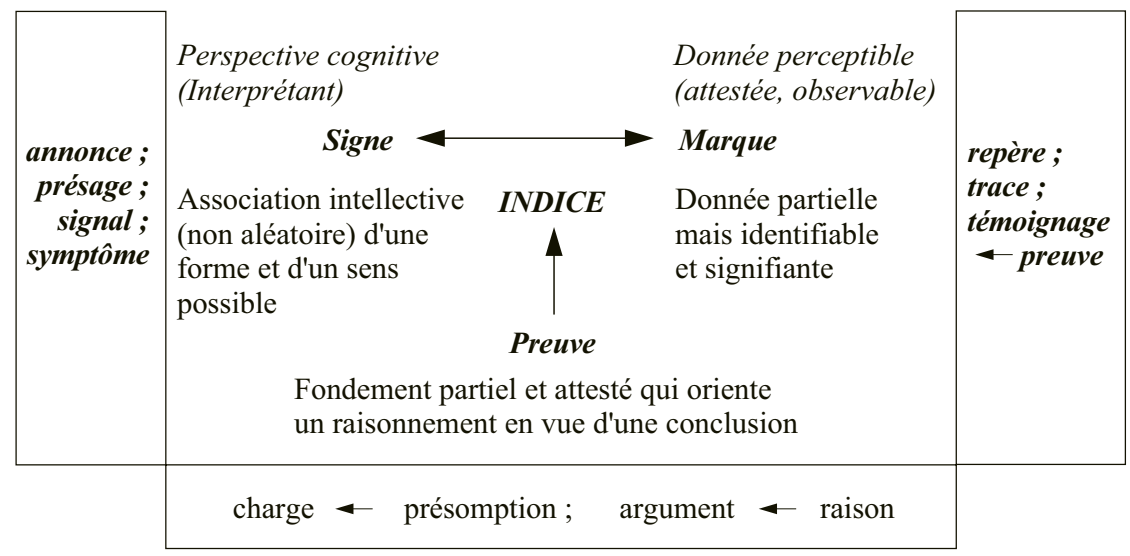

Figure 8 : «percevoir ou concevoir des indices» 


\section{Conclusion provisoire : les travaux qu'il reste à entreprendre}

Nous avons privilégié, au cours de ce travail sur l'unité indice, 1'outil DES du CRISCO et le guidage méthodologique de Jacques François (2007) ; et encore avons-nous été contraints d'édulcorer tel ou tel calcul (les distances entre cliques, par exemple), telle ou telle étape du raisonnement (par exemple le « profil dictionnairique » des entrées). Mais, outre ces raccourcis, nous avons également renoncé à introduire d'une part des extraits de corpus, d'autre part les collocations fondamentales, à savoir l'analyse du pluriel (les indices) et la structure $\mathrm{N}$ de $\mathrm{N}$ (des indices de crise), et enfin la propriété morphosémantique d'être un nom prédicatif (l'indice indique; ce mégot mal éteint est un indice à conserver) et transitif (l'indice indique quelque chose).

L'opposition spécifique / générique (y compris dans ses rapports compliqués avec l'hyperonymie et la procédure d'abstraction) ouvre, semble-t-il, une perspective assez prometteuse pour un nouvel examen comparatif des synonymes d'indice, des classements obtenus et du discours lexicographique (exemples, définitions et renvois analogiques). On sait bien en effet qu'un pneu laisse une trace, un pas une empreinte ou un coup une marque, mais que ni les uns ni les autres ne livrent directement ni signe ni indice. En revanche, les traces, les empreintes et les marques peuvent constituer des indices «précieux » comme dit le détective. En définitive, ce qui a retenu notre attention tout au long de ce travail exploratoire et sur quoi il nous faudra revenir, c'est la prise en compte du phénomène de « synonymicité » à travers cette conception d'un lexique dont les unités se rapprochent ou s'éloignent, au gré de divers calculs qui les hiérarchisent.

Enfin, Il nous semble que l'investigation lexicale présentée permet de réexaminer des interrogations qui étaient relativement confuses au début de notre travail. Ces questions concernent l'absence de certains «synonymes " d'indices dans les calculs de résultats du $D E S$, items pourtant attestés à travers la conjonction d'une opération de commutation et d'une reprise anaphorique, dans les discours, théoriques ou romanesques, que nous avons pu parcourir (hypothèses, détails, points, observations, par exemple). D'une certaine façon, nous sommes enclins à trouver un argument qui vérifie que l'anaphore nominale et la synonymie ne font pas bon ménage et que, décidément, la synonymie ne se réduit pas à la commutation en cotexte. 


\section{Indications bibliographiques}

Site du CRISCO : http ://www.crisco.unicaen.fr

DuBOIS, J., ([1992], 2006) : Le roman policier et la modernité, Paris : Armand Colin, pp. 123-131.

FRANÇOIS, J. (2003) : La prédication verbale et les cadres prédicatifs, LouvainParis, Éditions Peeters.

— (2005) : «Polysémie verbale et cadres participatifs : DEMANDER et ses synonymes », dans F. Lambert et H. Nølke, eds, La syntaxe au cœur de la grammaire, Mélanges Claude Muller, Presses Universitaires de Rennes, pp. 85-98.

—(2007) : Pour une cartographie de la polysémie verbale, Louvain-Paris : Peeters.

Todorov, T. (1971) : « Typologie du roman policier», Poétique de la prose, Paris : Seuil, pp. 55-65.

François, J., Manguin, J.-L., Eufe, R., Fesenmeier, L., Ozouf, C. \& SÉnÉCHAL, M. (2004) : Le Dictionnaire Electronique des Synonymes : un mode d'emploi à trois niveaux. Cahier du CRISCO 17, téléchargeable sur le site www.crisco.unicaen.fr

FRANÇOIS, J., ViCTORRI, B., \& MANGUin, J.-L. (2005) : «Polysémie adjectivale et synonymie, l'éventail des sens de CURIEUX étudié à l'aide des méthodes informatiques combinant un dictionnaire électronique de synonymes et des extraits de corpus », dans O. Soutet, dir., La polysémie, Paris, Presses de l'Université Paris Sorbonne : 175-188.

LOWE, R. (2007) : Introduction à la psychomécanique du langage, Laval, PUL.

MAnguin, J.-L., FranÇOIS, J., \& VictorRi, B., 2004, « Polysémie adjectivale et rection nominale : quand gros et gras sont synonymes », dans J. François, dir., L'adjectif en français et à travers les langues, Caen, Presses Universitaires de Caen : 521-540.

PICOCHE, J. (1986) : Structures sémantiques du lexique français, Paris, Nathan.

VICTORRI, B.\& FUCHS, C. (1996) : La polysémie : une construction dynamique du sens, Paris, Hermès.

\section{Dictionnaires}

[Rappelons que le DES recourt à Bailly, Bénac, Bertaud du Chazaud, Guilbert, Guizot, Lafaye et Robert. Les autres titres figurant ci-dessous ont été consultés par nos soins.]

BAILly, R. (1947) : Dictionnaire des synonymes, Paris, Larousse.

BÉNAC, H. (1956) : Dictionnaire des synonymes, Paris, Hachette.

BERTAUD DU CHAZAUd, H., ([1979] 2004) : Dictionnaire de synonymes et mots de sens voisin, Paris, Editions Le Robert \& Quarto Gallimard.

BRIO (2004) : Paris, Le Robert.

GUILBERT, L.et al. (1971): Grand Larousse de la langue française (GLLF), Paris, Larousse. 
Guizot, F. (1864 $)$ : Dictionnaire Universel des synonymes de la Langue Française, Paris, Didier.

LAFAYE, P.-B. (1858) : Dictionnaire des synonymes de la Langue Française, Paris, Hachette.

LEXIS, Larousse de la langue française (2002) : Paris, Larousse.

MEL'CUK, I., \& POLGUÈRE, A. (2007) : Lexique actif du français. L'apprentissage du vocabulaire fondé sur 20000 dérivations sémantiques et collocations du français, Bruxelles, De Boeck.

Picoche, J., \& Rolland, J.-C. (2002) : Dictionnaire du français usuel ; 15000 mots utiles en 442 articles, Bruxelles, De Boeck-Duculot.

REY, A. (2007) : le Nouveau Petit Robert (NPR), Paris, Éditions Le Robert.

ROBERT, P. (1975) : Dictionnaire alphabétique et analogique de la langue française. Les mots et les associations d'idées (le Grand Robert), Paris, Société du Nouveau Littré, Le Robert. 